\title{
If the IMPROVE-IT Trial Was Positive, as Reported, Why Did the FDA Denied Expanded Approval for Ezetimibe and Simvastatin? An Explanation of the Tipping Point Analysis
}

\author{
Emma P. Deloughery, $B S^{7}$ and Vinay Prasad, M.D. ${ }^{2}$ \\ 'Mayo Clinic School of Medicine, Rochester, MN, USA; ${ }^{2}$ Knight Cancer Institute, Oregon Health and Science University, Portland, OR, USA.
}

J Gen Intern Med 33(8):1213-4

DOI: $10.1007 / \mathrm{s} 11606-018-4498-3$

(c) Society of General Internal Medicine 2018

$I^{\prime \prime}$ n 2015, the Improved Reduction of Outcomes: Vytorin Efficacy International Trial (IMPROVE-IT) trial was published in the New England Journal of Medicine and claimed the addition of ezetimibe to simvastatin reduced the risk of cardiovascular events in patients with a previous cardiovascular event. ${ }^{1}$ The benefit in the trial was a $2 \%$ absolute or $6.4 \%$ relative risk reduction in cardiovascular events in the ezetimibe/simvastatin group as compared with simvastatin alone. This reduction was primarily driven by a reduction in non-fatal myocardial infarction and non-fatal stroke. According to the primary trial publication, IMPROVE-IT achieved its goal of showing a reduction of cardiovascular events in patients taking the ezetimibe/simvastatin combination, 'yet the U.S. Food \& Drug Administration (FDA) thought otherwise, and denied marketing approval for ezetimibe-simvastatin based on these data. ${ }^{2}$

Given the persistent difference between the academic record and the FDA's decision, the reasoning should be explored. The denial of the approval hinged on the small magnitude of event reduction with the ezetimibe/simvastatin combination, causing the results to be viewed as clinically insignificant by some experts. ${ }^{2}$ And, though some analyses of the trial argue that IMPROVE-IT's design and methodology resulted in a high degree of confidence in the results, due to low risk of bias and adequate statistical power, ${ }^{3}$ the FDA noted IMPROVE-IT had two key flaws: there was a large volume of missing data, and the researchers likely underestimated the rate of cardiovascular events when making assumptions about that missing data.

IMPROVE-IT had a high rate of discontinuation. Approximately $11 \%$ of study participants discontinued follow-up early for the primary endpoint of cardiovascular events. ${ }^{4}$ Around 5\% of study participants, more in the ezetimibe/simvastatin group,

Received March 26, 2018

Revised May 16, 2018

Accepted May 16, 2018

Published online June 4, 2018 discontinued at the end of the first year, while the median duration of the study was six years. Such early discontinuation had a greater impact on the study than later discontinuation due to greater missing time where a cardiovascular event could have occurred. Also, the observed rate of cardiovascular events was highest in the first year (13.5\% compared with $5.3-2.6 \%$ in subsequent years). ${ }^{4}$ Since most patients who discontinued did so in the first year, and most cardiovascular events occurred in the first year, then there is the possibility that some cardiovascular events were missed due to discontinuation. Such a possibility was adjusted for in the statistical analysis, where in order to generate a complete dataset for analysis, the rate of cardiovascular events in the missing data from the discontinued patients in the simvastatin group was estimated at 6.64 per 100 patient years based on data from prior studies.

In order to determine the robustness of the study's conclusions to the assumptions made when generating the missing data, both the researchers and the FDA performed a tipping point analysis. Tipping point analyses vary parameters until a result is "tipped" from statistical significance into non-significance, or vice versa. The more extreme the "tipping" parameters are from what would be expected, the more robust the conclusion. When varying the hazard rates for the missing time in the simvastatin and ezetimibe/simvastatin groups, the IMPROVE-IT researchers were able to "tip" their results into non-significance at an estimate of 6.64 events per 100 patient years for the missing data in the simvastatin group and 7.17 per 100 patient years for the ezetimibe/simvastatin group, creating a hazard ratio of 1.08 instead of the 0.94 generated using the randomly generated event estimates. Given that a hazard ratio of 1.08 would mean that more events occurred in the ezetimibe/ simvastatin group than in the simvastatin group, as opposed to the analysis which showed the opposite, this would seem to show that the conclusion of the superiority of the ezetimibe/ simvastatin combination to simvastatin alone was robust. However, in their analysis, the FDA thought the actual rate of cardiovascular events in the simvastatin group was likely to be higher due to the greater number of events that occurred in the first year of the study, when many of the participants discontinued. Using an estimate of 9.6 events per 100 patient years in the simvastatin group tipped the results into nonsignificance at a hazard ratio of 1.04 , with 9.98 events in the ezetimibe/simvastatin group. With increasing estimated rates of 
cardiovascular events in the simvastatin group, the tip into statistical non-significance became easier to obtain at hazard ratios ever closer to one. In the first year of the study, the cardiovascular event rate was 13.5 in both groups, and using that rate for the simvastatin missing data "tips" the conclusion at a hazard ratio of 1.01 , with only 13.64 events in the ezetimibe/ simvastatin group. That the result of the study is more easily "tipped" with higher - arguably more realistic scenarios - event rates indicates that the conclusion is less robust than as presented by the researchers. That is, given the uncertainty regarding the true event rate in the first year, the volume of missing data is too great to allow for a conclusion to be decisively drawn on the effects of ezetimibe/simvastatin as compared with simvastatin on the frequency of cardiovascular events.

It is easy to take a study at its word that its results are significant, but in the case of the IMPROVE-IT trial, we believe the FDA was right not to approve the expanded indication despite the purported efficacy of the ezetimibe/simvastatin combination. With the high rate of discontinuations and the greater cardiovascular event rates in the first year, the data is not robust to alternative assumptions regarding the missing data. This calls into question the significance of the results, and given the already small reported reduction in events with the ezetimibe/simvastatin combination, it seems more likely that ezetimibe/simvastatin's effect on cardiovascular events remains unknown and that further study is needed.

The difference between the FDA documents regarding ezetimibe/simvastatin and the journal publication also highlight the importance of drug regulators. Evidence appraisals by regulatory agencies can complement the important role of journals in the dissemination of clinical trial results.

Corresponding Author: Vinay Prasad, M.D.; Knight Cancer Institute Oregon Health and Science University, Portland, OR, USA (e-mail:prasad@ohsu.edu).

Funding Information Dr. Prasad is funded by the Laura and John Arnold Foundation.

\section{Compliance with Ethical Standards:}

Conflict of Interest: Dr Prasad is the author of Ending Medical Reversal, and receives royalties.

\section{REFERENCES}

1. Cannon CP, Blazing MA, Giugliano RP, et al. Ezetimibe Added to Statin Therapy after Acute Coronary Syndromes. N Engl J Med. 2015;372(25):2387-2397.

2. Brauser D. FDA advisors: reject secondary-prevention ezetimibe indication. News 2015. https://www.medscape.com/viewarticle/855958. Accessed Aug. 05, 2017.

3. Correia LC. Ezetimibe: Clinical and Scientific Meaning of the IMPROVE-IT Study. Arq Bras Cardiol. 2016;106(3):247-249.

4. FDA Briefing Document. Endocrinologic and Metabolic Drugs Advisory Committee (EMDAC). In: Committees A, ed. [Online]: U. S. Food \& Drug Administration; 2015.

5. FDA Briefing Document. Oncologic Drugs Advisory Committee Meeting (BLA: 125646): Tisagenlecleucel Novartis Pharmaceuticals Corporation. In: Committee ODA, ed: U.S. Food and Drug Administration 2017;39:46. 